\title{
Licenciatura en Derecho en los penales de Lima, bajo el sistema de educación a distancia
}

Willy Ramírez Chávarry*

*Abogado, Licenciado en Educación, Coordinador de la Facultad de Derecho y Ciencias Políticas de la Universidad Alas Peruanas. Tiene el Título de Magister en Enseñanza y Aprendizaje Abiertos y a Distancia, por la Universidad Nacional de Educación a Distancia (UNED), de Espańa. 


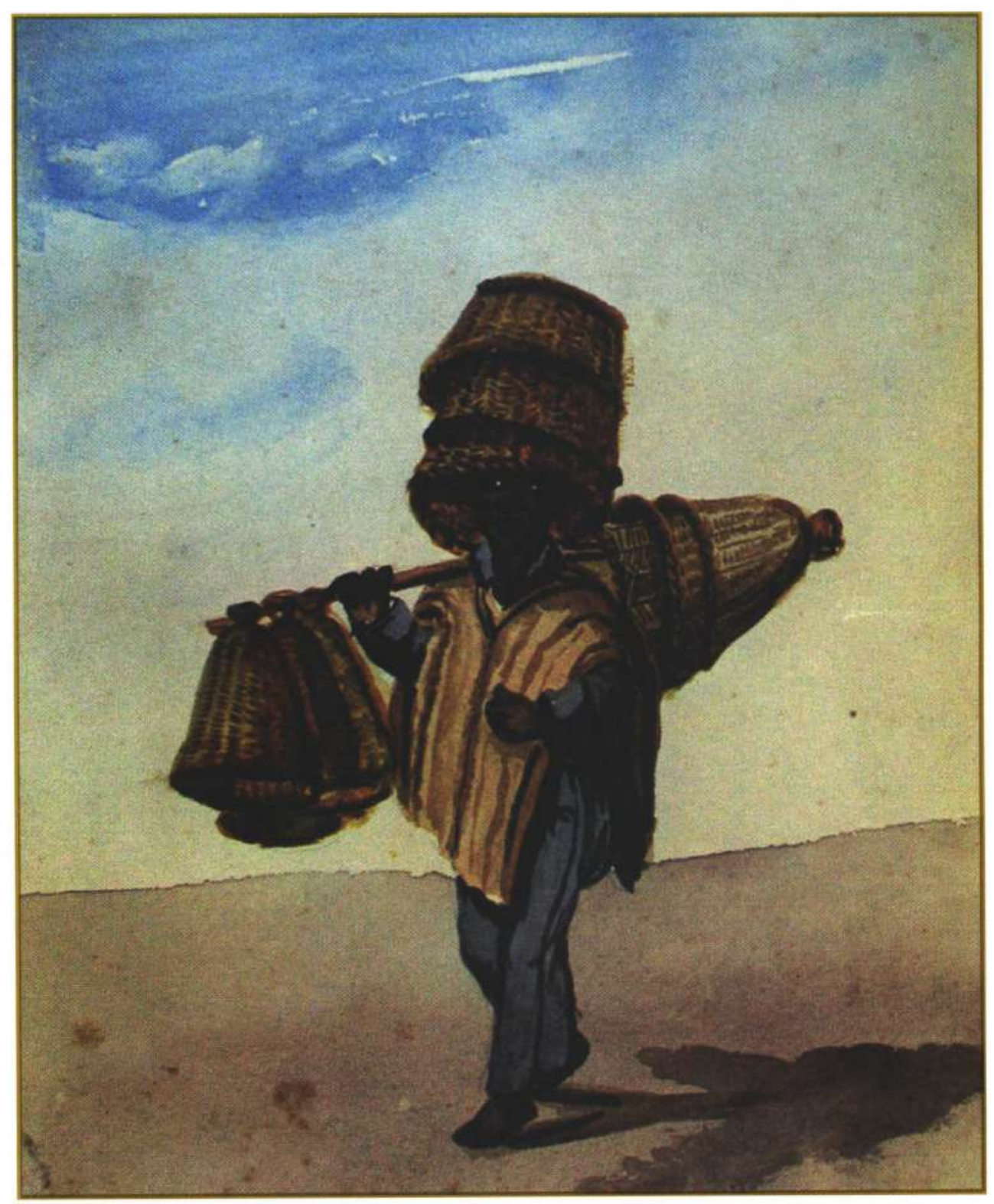

Vendedor de canastas. 


\section{INTRODUCCIÓN}

El presente trabajo trata el tema Licenciatura en Derecho en los Penales de Lima, bajo el sistema educativo abierto y a distancia de la Universidad Alas Peruanas. Para iniciar el tema explicamos la necesidad que tienen de formarse los ciudadanos peruanos que no pueden acceder a los sistemas de educación universitaria en la modalidad presencial tradicional, debido a su condición de internos. Luego destacamos los recursos y fortalezas con los que cuenta la Universidad Alas Peruanas (UAP) para hacerse cargo del requerimiento de estos estudiantes y abordar una política de educación universitaria a distancia que atienda esta demanda. Posteriormente, definimos las actividades que se deben realizar planteando la estructura organizacional, la creación del programa, el diseño y ejecución de la formación de profesores, tutores y personal de servicios en la modalidad a distancia, la construcción del material educativo y didáctico, así como las metodologías y procesos de aprendizaje, evaluación y retroalimentación de los alumnos y programas. Finalmente planteamos algunas consideraciones para la imple- mentación de nuevos programas de Educación a Distancia como licenciaturas y cursos de postgrado y extensión universitaria.

\section{JUSTIFICACIÓN}

La educación en el Perú se ha entendido como un importante factor para el desarrollo social, cultural, político y consecuentemente económico de nuestra nación. Sin embargo, en la actualidad la mayoría de internos de los establecimientos penitenciarios al culminar su condena, casi inexorablemente pasan a formar parte de la masa laboral desempleada por carencia de empleo o por la falta de calificación para el empleo existente. Esta realidad genera una situación problemática para el individuo y para sus familias, situación que se agrava porque el acceso a la educación superior se consigue solo en la modalidad tradicional de enseñanza presencial.

Por lo tanto, muchos ciudadanos que se encuentran purgando condena, simplemente no pueden acceder a la educación superior por encontrarse privados de su libertad. Adicionalmente, la mayoría de internos que por alguna razón no realizaron o interrumpieron sus estudios superiores universitarios, ven 
imposibilitadas sus aspiraciones de iniciar o continuar los mismos, ya que no están en condiciones de cubrir las exigencias de horarios, clases de asistencia obligatoria y a veces inflexibles programaciones académicas.

Ante esta realidad, en nuestro país, se requiere educar y formar integralmente a los internos de los establecimientos penitenciarios en una carrera de gran interés para ellos, a fin de que se cumpla el rol de la educación, entendido como un eje propulsor del desarrollo social, cultural, político y económico; asimismo, cumplir con los dispuesto por la Constitución política del Estado, que establece que es fin de la pena el reeducar y rehabilitar a los internos para reincorporarlo a la sociedad.

Las características de flexibilidad, autonomía de estudio, autoaprendizaje, innovación en sistemas educativos, uso intensivo de materiales educativos y didácticos, herramientas de comunicación, monitoreo, tutoría, eliminación de las barreras físicas, geográficas y de tiempo son las principales características por la que la educación superior abierta y a distancia se convierte en un sistema y modalidad viables y apropiados para el Perú, como lo han demostrado en países europeos la Open University del Reino Unido y la Universidad Nacional de Educación a Distancia de España, que incluso tienen alumnos y muchas experiencias en países latinoamericanos.

A través de un estudio de mercado sobre la preferencia de carreras profesionales de los internos de los establecimientos penitenciarios de Lima que se interesan por cursar estudios universitarios en la modalidad de educa- ción a distancia, se concluyó que los participantes prefieren la carrera de Derecho para iniciar estudios universitarios por primera vez o en el caso de alumnos que deseaban contar con otra licenciatura o querían acceder a esta licenciatura desde estudios superiores no universitarios. Este estudio fue realizado por la Dirección de Investigación de la Dirección Universitaria de Educación a Distancia de la Universidad Alas Peruanas mediante la aplicación de una encuesta que buscaba conocer si los participantes estaban interesados en seguir estudios en la modalidad a distancia, la carrera profesional que deseaban estudiar y si contaba con estudios superiores previos. Este estudio sirvió de base para la elección de la licenciatura que se desarrolla en el proyecto.

Este sistema implementado por la UAP permitirá tener abogados conocedores del sistema penitenciario peruano, que pueden dedicarse a este segmento del Derecho, asesorando a los internos de los establecimientos penitenciarios y sus familias. Muchos ejemplos más pueden servir para ilustrar el impacto altamente positivo en la sociedad y comunidad a través de la implementación del presente proyecto.

\section{CONTEXTUALIZACIÓN}

Los dos ejes institucionales sobre los que gira este proyecto, que busca reivindicar a un sector tradicionalmente marginado de la educación superior, son el Instituto Nacional Penitenciario (INPE) y la Universidad Alas Peruanas.

\section{El Instituto Nacional Penitenciario}

Es la institución encargada del sistema carcelario del país, organismo público descentra- 
lizado dependiente del Ministerio de Justicia. El INPE dirige y controla técnica y administrativamente el sistema penitenciario nacional. Entre sus funciones y atribuciones está la de celebrar convenios de cooperación con entidades públicas o privadas, nacionales o extranjeras.

En la actualidad existen 81 establecimientos penitenciarios que se encuentran en operación, distribuidos en ocho regiones. $\mathrm{La}$ población penitenciaria, según los datos oficiales a julio del año 2003, asciende a 28.948 internos, de los cuales 15.011 se encuentran en los establecimientos penitenciarios de la región Lima, es decir, el 51,86\%; siendo el hacinamiento uno de los problemas más agudos. La población penitenciaria a nivel nacional es de 26.928 varones $(93 \%)$ y 2.020 mujeres $(7 \%)$. En la región Lima la cifra es como sigue: 13.876 hombres y 1.135 mujeres privados de su libertad.

El grado de instrucción de los internos hasta el año 2002 ha sido relativamente bajo: analfabetos 5\%; Primaria incompleta 17,58\%; Primaria completa 11,59\%; Secundaria incompleta 27,06\%; Secundaria Completa 22,33\%; Superior no Universitaria 5,49\%; Superior Universitaria 4.6\%. En la región Lima, año 2002, los internos cursaron estudios distribuidos de la siguiente manera: Alfabetización: 98, Primaria: 623, Secundaria: 298, Ocupacional: 3,536 y Superior Universitaria 32. La educación ocupacional en el Perú está orientada a capacitar en un oficio específico y constituye enseñanza no reglada. En los establecimientos penitenciarios reciben capacitación ocupacional en los diversos talleres.
Es necesario mencionar que la Universidad Inca Garcilaso de la Vega brinda educación superior universitaria en uno de los establecimientos penitenciarios de Lima, en la modalidad semipresencial, ofertando la Licenciatura en Administración. Sin embargo la poca demanda o acogida que ha tenido ese Programa (32 estudiantes), se debe a que no es una carrera atractiva que los internos desean y a que el sistema aunque siendo semipresencial, es rígido en cuanto a las clases presenciales.

$\mathrm{Al}$ hacer un estudio sobre las preferencias vocacionales de los internos, tanto varones como mujeres, se obtuvo como respuesta un alto porcentaje interesado en cursar estudios de licenciatura en Derecho. El INPE cuenta con profesionales de diversas disciplinas, entre ellas: Educación y Derecho, en todos los establecimientos penitenciarios a nivel nacional, con mayor proporción en la región Lima, los cuales -una vez capacitados- realizan la labor de tutoría.

Todos los establecimientos penitenciarios cuentan con ambientes adecuados, como las aulas y oficinas administrativas para implementar un Centro Asociado o Unidad Descentralizada de la Universidad Alas Peruanas.

\section{La Universidad Alas Peruanas (UAP)}

El sistema de educación abierto y a distancia en el Perú, en el nivel superior universitario, ha sido también implementado por la Universidad Alas Peruanas, que cuenta con los recursos necesarios para su ejecución con calidad y eficiencia. Por ejemplo, contamos con 160 profesores capacitados en educación a Distancia, que siguieron el Primer 
Diplomado Internacional en Educación Abierta y a Distancia UAP- UNED (IUED). En la fase inicial de implementación del sistema de educación a distancia, se tiene planeado contar con 2,500 alumnos a nivel nacional. Actualmente venimos seleccionando y capacitando a los tutores de las Unidades Descentralizadas (Centros Asociados).

Todas las actividades de implementación del Sistema de Educación a Distancia UAP, están a cargo de la Dirección Universitaria de Educación a Distancia que cuenta con un staff de profesionales especializados en educación a distancia. Dentro de los recursos de infraestructura la UAP tiene diez locales universitarios en la sede de Lima y cinco locales en las sedes descentralizadas de provincias. Adicionalmente contamos con diez unidades descentralizadas a nivel nacional.

Hay bibliotecas en cada uno de sus locales, 1,200 computadoras personales en una red corporativa con conexión a Internet, sistema de servicios de intranet donde los alumnos, docentes, funcionarios y empleados pueden realizar consultas de carácter académico y administrativo, sistema de videoconferencia con líneas RDSI, sistemas de ayuda audiovisual, locales deportivos y cinco centros de formación práctica en el interior del país, especialmente para las licenciaturas que requieren tener contacto con las diferentes zonas geográficas.

El sistema de servicios de intranet implementado en el año 2001 le permite a los profesores alojar las páginas de sus asignaturas, publicar material bibliográfico digitalizado, material de autoevaluación, trabajos prácticos y recursos multimedia. Adicionalmente, los alumnos y profesores disponen de herramientas de mensajería para realizar consultas y debates fuera de clase. Los alumnos pueden consultar sus calificaciones, los reglamentos y manuales de estudios, avisos, calendarios académicos, horarios de clase y roles de evaluación, eventos, su estado de cuenta (pagos mensuales), entre otras informaciones de su interés. Los docentes pueden consultar sus horarios, obtener listados de alumnos, ingresar las calificaciones parciales y consultar los reglamentos, manuales, y otra información relevante para ellos.

Todos los docentes, empleados y alumnos disponen de una cuenta de correo y de acceso a Internet a través del servicio de acceso remoto del proveedor local "Infovía"; este servicio es proporcionado por la Universidad. La cuenta de usuario les permite ingresar también a los servicios de Intranet. Para todos estos servicios se ha implementado servidores académicos y administrativos de alta performance con procesamiento simétrico y redes de alta disponibilidad.

Como medios de apoyo, en el desarrollo de educación a distancia se cuenta con un canal de televisión y de una estación de radio con alcance nacional.

En efecto, el canal 51 UHF de señal abierta ha sido adquirido por la Universidad Alas Peruanas en 1999 y actualmente difunde programas de noticias, entrevistas y debates relacionados con la actualidad nacional y las actividades académicas y de extensión de la UAP. Asimismo, la Facultad de Ciencias de la Comunicación se encuentra trabajando en la elaboración de videos educativos. 
Los programas de televisión y videos educativos son un recurso sumamente importante en el sistema de educación a distancia y es utilizado, por las más importantes universidades con educación a distancia en el mundo. Esto constituye una fortaleza para la implementación de este proyecto.

Del mismo modo los programas de radio y casetes educativos constituyen un medio de ayuda más de las veces imprescindible para la formación de estudiantes, más aun en la modalidad de enseñanza abierta y a Distancia, permitiendo que los alumnos puedan recibir instrucción y participar en el proceso de enseñanza utilizando este medio, sin tener que desplazarse hasta las instalaciones de la Universidad. En este sentido el contar con la estación de radio representa de igual modo una fortaleza para la implementación de este proyecto.

Siendo el material escrito el medio de mayor relevancia en un proceso de enseñanza a distancia, es preciso contar con la capacidad de impresión que soporte la utilización adecuada en este medio. Es por esto que se ha adquirido en Alemania un sistema de imprenta de última generación con capacidad de imprimir todo tipo de textos y en tiempos muy cortos.

Otro factor importante para el desarrollo del sistema de educación en la modalidad abierta y a distancia es la incorporación de estudiantes en todo el territorio nacional por parte de la Universidad Alas Peruanas, ya que está cuenta con infraestructuras educativas de educación inicial, primaria y secundaria en cuatro ciudades y con institutos superiores tecnológicos en cinco ciudades, todas en el interior del país.

Adicionalmente se han establecido convenios de cooperación con decenas de centros educativos de nivel escolar y con institutos tecnológicos y pedagógicos en ciudades y centros poblados de provincias. Se cuenta también con convenios de cooperación con gobiernos locales y organizaciones cooperativas, así como del sector empresarial y productivo en estas localidades.

Los docentes requeridos para la implementación del sistema de enseñanza en la modalidad a distancia están garantizados con la plana docente actual, es decir, los docentes que ahora brindan enseñanza en el sistema presencial seguirán siendo entrenados adecuadamente para integrar la plana docente en la educación a distancia.

Con respecto a la tutoría, se cuenta con un número razonable de futuros tutores en los profesores de los centros educativos de nivel superior funcionando a nivel nacional y en otras instituciones de cada localidad, quienes con la capacitación y el entrenamiento que recibirán pueden hacerse cargo de las funciones asignadas a la tutorización y monitoreo de los estudiantes.

Tanto la actividad docente como la tutoría representan una altísima relevancia en el sistema de educación a distancia, debido a ello en el proyecto se tiene especial consideración en la selección, formación y evaluación continua de los profesores y tutores, para garantizar la eficiencia y calidad del proceso de enseñanza y formación que se requiere. 
Por lo expuesto, el factor en el que se trabajará con mucha fuerza en este proyecto es la formación del capital humano, bajo el sistema de educación abierta y a distancia, dándole a muchos ciudadanos la oportunidad que actualmente no brinda el sistema educativo tradicional.

\section{METODOLOGÍA Y PLAN DE ACTIVI- DADES}

Implementación de la dirección del programa de atención en establecimientos penitenciarios.

La creación de esta dirección es el primer paso que inicia la implementación de los programas a distancia en la Universidad Alas Peruanas dirigidos a los establecimientos penitenciarios, esta dirección será la encargada de desarrollar las actividades del presente proyecto y deberá contar con los recursos necesarios para alcanzar los objetivos propuestos.

\section{Creación de la Dirección del Programa de} Atención en Establecimientos Penitenciarios

Mediante Resolución Rectoral se deberá crear la Dirección del Programa de Atención en Centros Penitenciarios con las funciones y estructura organizacional que se definen en este trabajo.

Nombramiento del director de la
Dirección del Programa de Atención en Establecimientos Penitenciarios

Mediante Resolución Rectoral se decretará el nombramiento del Director que será el responsable de la Dirección que se acaba de crear y este deberá formar su equipo de trabajo en base a las funciones y perfiles que en este capítulo se indican.

\section{Estructura organizacional de la}

\section{Universidad Alas Peruanas}

Es preciso definir la ubicación de ésta dentro de la estructura organizacional de la Universidad, así como los modelos de gestión académica.

La Dirección del Programa de Atención en Establecimientos Penitenciarioses un órgano que reporta a la Dirección Universitaria de Educación a Distancia y mantiene coordinación directa con las Facultades, Escuela de Postgrado, Dirección de Educación Continua, entre otros.

Sistema de gestión Administrativo y Académico, la estructura organizacional de la Dirección del Programa de Atención en Establecimientos Penitenciarios se puede apreciar en el siguiente organigrama:

El director del Programa de Atención en Establecimientos Penitenciarios reporta al director de la Dirección Universitaria de

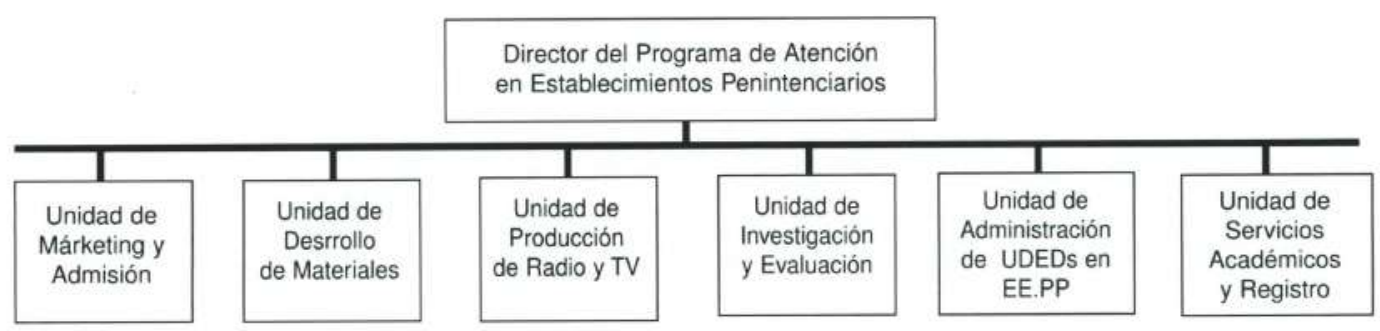


Educación a Distancia y administra la dirección cumpliendo y haciendo cumplir las funciones asignadas de definir las políticas y estrategias de educación a distancia en establecimientos penitenciarios, incorporar nuevas tecnologías educativas en esa materia, elaborar el plan estratégico y planes operativos del área, alcanzar los objetivos y metas propuestos en ellos. El perfil que se requiere para este puesto es poseer estudios y formación previa en educación a distancia, deberá poseer el grado de doctor y experiencia no menor de cuatro ańos en puestos de la responsabilidad y envergadura que el propuesto.

El Jefe de la Unidad de Márketing y Admisión es el encargado de diseñar e implementar el plan de márketing y el márketing mix. Implementar las estrategias de publicidad, promoción y la admisión de los alumnos a la licenciatura propuesta, responsable de la creación e implementación de estrategias de posicionamiento y captación de alumnos dentro de los establecimientos penitenciarios. El perfil para este puesto es ser licenciado en administración o márketing, con postgrado y especialización en marketing y con una experiencia no menor de cuatro años en márketing educativo. Coordina con la Dirección Adjunta de Márketing y Admisión de la Dirección Universitaria de Educación a Distancia y con las autoridades penitenciarias correspondientes.

La Unidad de Desarrollo de Materiales está compuesta por los docentes responsables de elaborar y actualizar el material educativo en los diferentes medios por utilizar. El equipo de esta área debe cumplir el siguiente perfil: ser docentes del curso que corresponda, con formación en educación y experiencia en la elaboración de material educativo para la modalidad a distancia. Coordina con la Dirección Adjunta de Desarrollo de Materiales de la Dirección Universitaria de Educación a Distancia y con las autoridades penitenciarias correspondientes.

La Unidad de Producción de Radio y Televisión es la encargada de producir los medios audiovisuales, atendiendo los requerimientos de los docentes y trabajando con ellos en la elaboración de casetes, videos y programas de radio y televisión educativos sobre las asignaturas dictadas. Asimismo cubrir los eventos académicos, científicos o tecnológicos que cuenten con la aprobación de la dirección. El perfil de esta área es ser catedrático con formación y experiencia en la elaboración de material audiovisual educativo, tanto en radio como en televisión. Coordina con la Dirección Adjunta de Producción de Radio y Televisión de la Dirección Universitaria de Educación a Distancia y con las autoridades penitenciarias correspondientes.

La Unidad de Investigación y Evaluación se encarga de incorporar las nuevas tecnologías y metodologías de enseñanza aprendizaje en la modalidad abierta y a distancia así como a evaluar la calidad de los materiales educativos desarrollados en la dirección y los procesos y procedimientos educativos y de tutoría. El perfil para este puesto es poseer formación y experiencia en sistemas de enseñanza y aprendizaje a distancia y haber trabajado en evaluaciones de material educativo. Coordina con la Dirección Adjunta de Investigación y Evaluación de la Dirección Universitaria de Educación a Distancia y con las autoridades penitenciarias correspondientes. 
La Unidad de Administración de Unidades Descentralizadas en los Establecimientos Penitenciarios es la encargada de administrar, recibir los requerimientos, coordinar y atender a las unidades descentralizadas instaladas en los establecimientos penitenciarios. El perfil de este puesto es ser licenciado en administración con estudios de postgrado en gestión de la educación superior y haber desempeñado puestos de la responsabilidad y envergadura similares. Coordina con la Dirección Adjunta de Administración de Unidades Descentralizadas de la Dirección Universitaria de Educación a Distancia y con las autoridades penitenciarias correspondientes.

La Unidad de Servicios Académicos y Registro es la encargada del registro, seguimiento y control de los alumnos de las diferentes licenciaturas. El perfil para este puesto es ser licenciado, preferentemente con estudios de post grado y experiencia mínima de cuatro años en trabajos similares. Coordina con la Dirección Adjunta de Servicios Académicos y Registro Central de Educación a Distancia de la Dirección Universitaria de Educación a Distancia y con las autoridades penitenciarias correspondientes.

Establecimiento de Convenio de Cooperación con el Ministerio de Justicia e Instituto Nacional Penitenciario

Este proyecto exige la actuación de las autoridades del Ministerio de Justicia, en general, y del Instituto Nacional Penitenciario, en específico; puesto que es este sector y esta institución los responsables del Sistema Penitenciario Nacional.
Se requiere cooperación técnica en tres aspectos fundamentales en la implementación de este programa:

Infraestructura: los establecimientos penitenciarios deben brindar todas las facilidades en la implementación de las unidades descentralizadas, destinando ambientes adecuados para la atención a los alumnos, tales como: aulas, oficina administrativa, biblioteca, laboratorio de informática.

Tutores: los tutores son propuestos por la Dirección del Establecimiento Penitenciario. Son seleccionados y capacitados por la Dirección del Programa. Es importante señalar que en cada establecimiento penitenciario se cuenta con un equipo de profesionales de las ciencias sociales y jurídicas que podrían realizar esta labor.

Sistema logístico: las autoridades penitenciarias deberán brindar todas las facilidades para el ingreso de profesionales y de material educativo a los establecimientos penitenciarios. Asimismo para la realización de actividades extracurriculares.

\section{Creación de las Unidades Descentralizadas} de Tutoría y Atención al Alumno en los Establecimientos Penitenciarios

Las unidades descentralizadas de tutoría y atención del alumno son un componente fundamental en el sistema de educación abierto y a distancia a implantar, estos representan las unidades de enlace entre la universidad y los alumnos, quienes no tienen la posibilidad de acudir a los centros de tutoría fuera de los establecimientos penitenciarios. 
Estas unidades contarán con la infraestructura que permita desarrollar las actividades de tutoría y evaluación a los alumnos, así como los recursos de biblioteca, laboratorios de informática, cafetería, aulas y despachos de tutoría para soportar los requerimientos en función al número de estudiantes.

Es importante que la creación y funcionamiento de estas unidades cuente con el apoyo de las autoridades penitenciarias, población penitenciaria, organismos no gubernamentales, así como a las empresas e instituciones que pueden convertirse en colaboradores y patrocinadores de estos.

La estructura organizacional básica de las unidades descentralizadas se puede apreciar en el siguiente organigrama:

El director de la unidad reporta a la Dirección del Programa de Atención en Establecimientos Penitenciarios de la
Dirección Universitaria de Educación a Distancia ubicada en la sede central y coordina activamente con las unidades de Márketing y Admisión, Administración y de Desarrollo de Materiales. El perfil requerido para este puesto es ser licenciado en educación, preferentemente con grado de doctor, haber trabajado cuatro años como mínimo dirigiendo instituciones educativas. Serán sus funciones: dirigir la unidad, establecer convenios con instituciones locales y administrar los existentes, planificar las actividades de la unidad y controlar el desarrollo de las actividades académicas y administrativas de la Unidad.

La Unidad de Biblioteca y Librería se encarga de atender a los alumnos en el material bibliográfico. Existe coordinación con la biblioteca de la sede central. El perfil para este puesto es tener una experiencia no menor a dos años en puestos similares y tener estudios superiores.

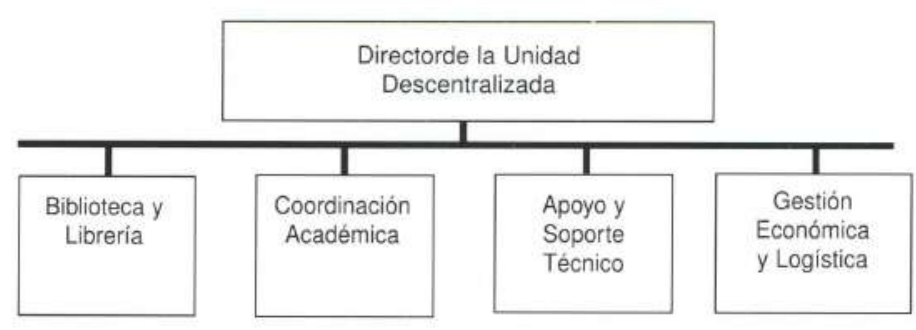


La unidad de coordinación académica se encarga de definir la plana de profesores tutores, calendarios, horarios y gestión de las tutorías. Se encarga asimismo de las matrículas y atención de los alumnos. Coordina y atiende a los profesores tutores, supervisa su trabajo y es el nexo entre estos con los profesores de la sede central. El perfil de este puesto es ser licenciado de preferencia con el grado de doctor, poseer formación en gestión de instituciones educativas y haber trabajado como mínimo cuatro años en puestos similares.

La Unidad de Apoyo y Soporte Técnico se encarga de administrar y mantener operativo los recursos técnicos, informáticos y los recursos adicionales con los que cuente la unidad descentralizada. Existe coordinación con la Dirección de Informática de la sede central. Debe tener estudios superiores y experiencia mínima de dos años en puestos similares.

La Unidad de Gestión Económica y Logística, se encarga de administrar y reportar a las áreas de la sede central de Contabilidad, Planificación y Logística. Gestiona también con la logística de la Unidad Descentralizada. El perfil de este puesto es ser licenciado en Contabilidad o Administración, preferentemente con estudios de postgrado y una experiencia mínima de tres años en puestos similares.

En la fase inicial de la implementación del programa, el director de la Unidad Descentralizada desempeñará las funciones de la Coordinación Académica y de la Unidad de Gestión Económica y Logística.

\section{SISTEMA LOGÍSTICO Y PROCESOS DE SOPORTE}

Se requiere definir los procesos logísticos y de soporte necesarios para que el resultado de los procesos principales (elaboración de materiales, formación de profesores y alumnos, entre otros) nos lleven al cumplimiento de los objetivos propuestos.

Dentro de estos procesos podemos mencionar los siguientes: proceso logístico, elaboración y distribución de materiales en forma oportuna, y con los estándares que la unidad responsable de materiales ha establecido; atención de las compras y el manejo de la relación con proveedores; trabajos de mantenimiento y servicios de los locales.

\section{Proceso de mercadeo}

Sistema de difusión y posicionamiento con estrategias que permitan captar la atención de los estudiantes y ofrecer adecuadamente el servicio que estos están requiriendo.

Elaboración de un plan de márketing con campañas de acercamiento de los estudiantes, empresas e instituciones estatales para comprometerlos concretamente, en términos de convenios, para el apoyo material y político requerido.

Realización de certámenes nacionales e internacionales, seminarios y congresos con la presencia de autoridades académicas y penitenciarias.

Realización de certámenes específicos para las autoridades penitenciarias, de gobiernos locales y del gobierno central. 


\section{Proceso de admisión, Matricula y trámites}

Adecuado proceso de difusión con medios de comunicación de acuerdo a cada establecimiento penitenciario.

Sistema de matrícula ágil y accesible a todos los estudiantes.

Sistemas de cobranza y recaudación ágiles y modernos, adecuados a la condición de internos de los establecimientos penitenciarios.

Sistema de trámite documentario ágil y de procedimientos sencillos para el alumno.

Definición de calendarios y cronogramas que incluyan todas las actividades para el alumno, así como un medio de difusión que permita que todos los alumnos lo conozcan.

\section{Proceso de evaluación y reporte de cali- ficaciones}

Sistemas de aplicación de exámenes rígidos en cuanto a su formalidad pero al mismo tiempo no traumatizantes para los alumnos.

Sistema de calificación y reporte de resultados ágil que permita al alumno conocer sus notas en un plazo prudencial de tiempo.

Sistema que permita la retroalimentación de la eficiencia de las pruebas y de las dificultades que tienen los alumnos, así como la forma en la que se pueden mejorar continuamente.

Proceso de culminación y cierre de un semestre.
Cumplimiento de calendarios cronogramas establecidos.

Emisión de actas finales y refrendos oficiales en plazos adecuados.

Descripción de Procesos

PROGRAMA DE ENTRENAMIENTO DE PROFESORES, TUTORES Y PERSONAL ADMINISTRATIVO

\section{Capacitación de profesores}

El entrenamiento y capacitación del personal docente se realizó en dos etapas que involucran trabajo colaborativo y metodologías de aprendizaje a distancia. La primera etapa consistió en preparar a un grupo de cuatro docentes en cursos de nivel internacional sobre la enseñanza y aprendizaje abiertos y a distancia, que incluyan en su propia formación asignaturas dictadas en esta modalidad.

La segunda etapa corresponde a la preparación de un grupo de cuarenta profesores quienes atenderán la demanda de las asignaturas involucradas en la licenciatura a implantar. Esta formación estuvo a cargo de los docentes que recibieron el entrenamiento internacional, en esta etapa también se incluirá asignaturas dictadas a distancia. Esta etapa será replicada en el segundo año de aplicación del proyecto para 120 profesores y contará con las mismas características que el anterior.

De esta manera los docentes participarán primero en su propia formación en la modalidad abierta y a distancia para que posteriormente, habiendo vivido esta experiencia previa en esta modalidad, puedan preparar a los alumnos que vayan a participar en los programas a implantar. 
El entrenamiento de la primera etapa estuvo basado en la Maestría en Enseñanza Abierta y a Distancia impartido por la Universidad Nacional de Educación a Distancia y consta de preparación en Inmersión en una Plataforma Virtual de aprendizaje, Fundamentos de la Educación a Distancia , El Aprendizaje a Distancia, La Docencia en la Enseñanza y Aprendizaje Abiertos y a Distancia, La comunicación didáctica, Los medios convencionales en la Enseñanza y Aprendizaje Abiertos y a Distancia, Nuevas Tecnologías de la Información y de la Comunicación, La evaluación de los aprendizajes en la enseñanza a distancia, Planificación y organización de sistemas de Enseñanza y Aprendizaje Abiertos y a Distancia -Perspectiva internacional, La calidad de la educación a distancia, La evaluación institucional y de programas, creación de cursos virtuales.

El entrenamiento de la segunda etapa se realizó sobre cursos específicos en: Fundamentos de Educación a Distancia, Docencia en la Enseñanza y Aprendizaje Abiertos y a Distancia y en La Elaboración de Material Didáctico: Guías y Unidades Didácticas.

Todos los docentes recibieron un curso sobre ofimática, herramientas de Internet y manejo de la plataforma de Intranet. De esta manera, podrán utilizar los medios tecnológicos disponibles dentro del desarrollo de su cátedra.

Los criterios de selección de profesores son la relación de su especialidad con las asignaturas, deberán contar preferentemente con estudios de postgrado y experiencia docente previa.

\section{Capacitación de tutores}

Los profesores tutores serán seleccionados en base currículum, años de experiencia docente, experiencia profesional, así como también se considerará adecuado, siguiendo las premisas anteriores, seleccionar a aquellos que ejercen en el nivel medio a fin de aprovechar sus expectativas de desarrollo profesional y su incursión a un sistema universitario sin el contacto previo del modelo tradicional, si no más bien directamente al modelo a distancia.

Los profesores tutores serán propuestos por la Dirección de cada Centro Penitenciario y entrenados por la Dirección Adjunta de Investigación y Evaluación de la Dirección Universitaria de Educación a Distancia. Deberán recibir una orientación sobre el sistema educativo planteado por la Universidad Alas Peruanas, conocer sus funciones, sus objetivos y contar con un manual que le permita iniciar adecuadamente sus actividades.

Recibirán también todos los tutores un curso de ofimática, herramientas de Internet y manejo de la plataforma de Intranet. De esta manera, podrán utilizar los medios tecnológicos disponibles dentro del desarrollo de sus actividades. El número de tutores a capacitar se encuentra en el cuadro de proyección de recursos de personal.

En la modalidad a distancia la tutoría es un elemento fundamental debido a que permite establecer contacto con el alumno, inducirlo correctamente al modelo de educación a distancia y asimismo permitir que los alumnos se relacionen entre ellos, se conozcan y establez- 
can lazos de sociabilización que contribuirá a minimizar el efecto de estudio solitario que es característica del modelo de educación que han elegido.

Se impartirá una tutoría introductoria o clase de orientación obligatoria para los alumnos en la que se le presentará el modelo de educación a distancia de la Universidad Alas Peruanas, se le explicará la metodología a emplear, los recursos con los que cuentan y la forma correcta de utilizarlos. Posteriormente la asistencia de los alumnos a la tutoría es opcional y voluntaria.

\section{Capacitación del personal administrativo}

Es importante entrenar al personal administrativo que laborará tanto en la sede central como en las unidades descentralizadas dentro de los establecimientos penitenciarios, a fin de establecer un ambiente de trabajo que cuente con una coordinación y comunicación adecuada y que esto redunde en los servicios prestados al alumno. Para esto la capacitación consistirá en el curso de introducción al modelo de enseñanza a distancia y un curso sobre la calidad de servicios a cargo de la Escuela de Administración.

\section{ELABORACIÓN DE MATERIALES}

La elaboración de los materiales es el componente más importante y esencial en el sistema propuesto, debido a que estos reemplazan al profesor de la educación tradicional en la que se exige la presencia de alumnos.

Los textos, guías y unidades didácticas, medios de ayuda de audio, videos, herramientas computacionales y telemáticas, serán desa- rrolladas por la unidad de elaboración de materiales conformando equipos multidisciplinarios que trabajarán conjuntamente con los profesores del curso.

Se realizarán simulaciones de utilización de los materiales por parte de los alumnos estableciendo mecanismos de retroalimentación y mejora antes de que estos sean distribuidos, atendiendo a las observaciones de los alumnos producto del uso simulado los materiales. La Dirección Adjunta de Elaboración de Material Didáctico de la Dirección Universitaria de Educación a Distancia es la encargada de dar el visto bueno para la culminación del material. En la elaboración de los materiales habrá estrecha coordinación y monitoreo con otras áreas de la universidad a fin de aprovechar todos los recursos con los que se cuentan de manera corporativa. Para la elaboración de material escrito, por ejemplo, se trabajará con las facultades correspondientes, para la elaboración de material informático como CD-ROM y páginas Web se coordinará con la Dirección de Informática y para la elaboración de videos, casetes, radio y TV se coordinará con la Dirección de Medios de Comunicación.

Cada asignatura contará con un material mínimo: guías didácticas, guía de calendarización, unidades didácticas, textos de consulta, página Web y documentos digitalizados, herramientas de correo $y$ foros de debate. Asimismo, con un material opcional: videos, casetes, CD-ROM.

Las guías didácticas representan el documento que orienta al alumno en su estudio, facilitando el trabajo autónomo de éste 
mediante el acercamiento con el material didáctico de la asignatura. Una buena guía didáctica debe contener: presentación de la asignatura y del equipo docente, introducción de la asignatura, objetivos, prerrequisitos, materiales, contenido, orientaciones bibliográficas, orientaciones para el estudio, actividades, glosario, tutoría y evaluación.

Las unidades didácticas representan un conjunto integrado, organizado y secuencial de los elementos básicos que representan el proceso de enseñanza-aprendizaje. Estas deben tener una introducción y orientaciones para el estudio, explicación de la utilidad y credibilidad de la información que en ella se vierte, detalles, secuencia entre unidades, estructura de contenidos, resumen, bibliografía y actividades.

Existen métodos bastante estudiados y elaborados para desarrollar las guías y unidades didácticas, debido a su importancia en el proceso de enseñanza y aprendizaje a distancia; requieren de personal altamente calificado para su implementación por lo que en la formación de personal docente se ha contemplado con especial cuidado este importante tema.

\section{METODOLOGÍA DE ENSEÑANZA Y EVALUACIÓN}

La metodología de enseñanza está basada en la preparación individual y autónoma del alumno con base al material educativo que la universidad le imparte. La autonomía en el estudio y el autoaprendizaje son los factores básicos de esta metodología, en la cual el alumno estudia en el ritmo y profundidad que sus posibilidades se lo permiten. Es decir un alumno puede matricularse en todas las asig- naturas delplan de estudios en un semestre o elegir sólo algunas de ellas.

La complejidad de las asignaturas cursadas se basan en la naturaleza de cada una de ellas, donde los materiales de estudio elaborados y distribuidos por la universidad aseguran un método de estudio que le permitirá al alumno aprender las materias de igual o mejor manera que el método tradicional, siendo su única limitante el tiempo que disponga para realizar su autoestudio.

Los requisitos para iniciar los estudios universitarios de la licenciatura a implantar es haber culminado la educación secundaria, presentando los certificados de estudios y la documentación personal de identificación. Los alumnos a incorporarse a este sistema se pueden clasificar en tres tipos. En primer lugar los alumnos que han culminado sus estudios secundarios hace poco tiempo (uno a tres años) y en su localidad no hay universidad; en segundo lugar alumnos que han culminado sus estudios secundarios hace más de cuatro años y no realizaron ningún estudio superior previo y finalmente en tercer lugar estudiantes que cuentan con estudios superiores previos. En todos los casos es imposible que asistan a una universidad tradicional, debido a su condición de internos de los establecimientos penitenciarios. Las vacantes serán cubiertas por los alumnos que aprueben la admisión en estricto orden de méritos

En este sentido el recurso más importante en la metodología planteada son los materiales de estudio y las actividades de tutoría. El material que todo alumno recibirá o tendrá acceso consta de guías didácticas, calendariza- 
ción académica, horarios de tutoría, rol de exámenes, unidades didácticas, textos de consulta, página Web y documentos digitalizados, herramientas de correo y foros de debate. Asimismo contará con material opcional como videos, casetes, CD-ROM, y otros.

Otro aspecto importante en la metodología son las tutorías que permitirán a los alumnos aclarar sus dudas, así como a recibir motivación y apoyo moral imprescindibles para perseverar hasta conseguir el objetivo de culminar la asignatura y la carrera. En función al número de alumnos matriculados por curso en cada unidad descentralizada, se impartirán tutorías de asistencia voluntaria de una o dos horas semanales, en función del número de créditos de la asignatura, programándose estas en coordinación con las autoridades penitenciarias.

El plan de estudios se estructura en base a cursos obligatorios y cursos electivos, con una distribución de créditos definidos por la Facultad a través del currículo, el número total de créditos entre cursos obligatorios y electivos es de 250 , distribuidos en 12 semestres académicos. Los alumnos deberán cursar y aprobar la totalidad de los cursos obligatorios y los cursos electivos necesarios para completar el creditaje normado.

Los cursos de formación teóricos y teóricoprácticos se desarrollarán con los materiales indicados en este capítulo. Los cursos netamente prácticos y las prácticas profesionales deberán ser realizados obligatoriamente por los alumnos, contando para ello con una oferta propia de la Unidad Descentralizada a través de empresas del sector privado y público con las que se han establecido convenios y en las propias instituciones penitenciarias. Esta actividad es evaluada por los docentes de la sede central.

Se ha incluido en la metodología una tutoría introductoria o seminario de orientación obligatorio para los alumnos en los que conocerán de primera mano el modelo de educación a distancia de la Universidad Alas Peruanas, la metodología que utilizarán y los recursos con los que cuentan para desarrollar sus actividades en la unidad descentralizada. Este primer contacto les permitirá aclarar las dudas o las ideas que tenían previamente del programa, la carrera elegida y la metodología en la que están inmersos.

De esta manera se promueve la asistencia de los alumnos a la unidad descentralizada, lo que les permitirá relacionarse entre ellos, conocerse y formar grupos de estudio o de entretenimiento, sin duda este será un elemento de sociabilización que contribuirá a minimizar el efecto de estudio aislado que es característica del modelo de educación que han elegido.

En cuanto a la metodología de evaluación, ésta se realiza en exámenes escritos sobre las tópicos expuestos en las unidades didácticas y libros de texto, se verificará el logro de los objetivos propuestos en cada asignatura. Los exámenes serán elaborados por los profesores de cada asignatura y serán discutidos previamente por equipos docentes de la especialidad y de la asignatura en cuestión.

El procedimiento de evaluación consiste en aplicar dos exámenes presenciales por semes- 
tre, el primero en la novena semana y el segundo en la semana decimoséptima. Se dispone de un examen sustitutorio que puede reemplazar a la nota más baja obtenida en cualquiera de los dos exámenes anteriores y este se aplica en la decimoctava semana. El sistema de calificación es vigesimal con calificativos que van de cero a veinte.

Todos los exámenes son obligatoriamente presenciales, estarán programados en el calendario académico y los alumnos conocerán las fechas de estos desde el inicio de las actividades lectivas del semestres. La aplicación de los exámenes se realizará indefectiblemente en las fechas, horarios y duración establecidos en el calendario académico. Las características particulares del examen dependerán de la naturaleza de la asignatura y estarán consignadas en su respectiva guía didáctica. Los exámenes serán calificados por los docentes de la sede central.

La evaluación escrita presencial será utilizando principalmente pruebas de ensayo, pruebas prácticas de laboratorio y pruebas objetivas, dependiendo del tipo y naturaleza de cada asignatura. El alumno recibirá sus calificaciones por correo electrónico y en la unidad descentralizada en un plazo máximo de 15 días de aplicado el examen y a través de correo regular en un plazo no mayor de 30 días de aplicado el examen. Basándonos en la metodología de enseñanza y aprendizaje abierto y a distancia propuesta podemos definir los componentes principales de este modelo:

El alumno: elemento fundamental en toda actividad educativa, el proceso de enseñanza y las actividades educativas deben centrarse en él. Los materiales educativos deben cumplir con sus exigencias y expectativas, caracterizándose estos, según nuestro modelo, por ser personas adultas con deseos de aprender y formarse profesionalmente.

El docente: las características más importantes del docente son su formación, capacidades y actitudes. En ese sentido en este proyecto se plantea un conjunto de estrategias y acciones para formar los docentes requeridos para el programa planteado. En este elemento tenemos a su vez a dos protagonistas que desarrollan simultáneamente con el alumno el proceso educativo. Los profesores encargados de desarrollar y actualizar las asignaturas y materiales, y los tutores como facilitadores del autoestudio de los alumnos.

La comunicación: elemento fundamental en cualquier modelo de enseñaza, más aun en la modalidad a distancia. Se necesita definir los canales de comunicación a fin del que los emisores (docente, tutores) lleguen a entregar nítidamente su mensaje a los receptores (alumnos). Está comprobado que un de los principales problemas de los estudiantes por modalidades a distancia y motivo de su deserción es la soledad y el alejamiento del profesor y de sus compañeros. Esta es sin duda una necesidad de comunicación. Los canales y sistemas de comunicación a utilizar, como ya se ha expuesto, en la implementación del sistema de educación a distancia en la Universidad Alas Peruanas serán el material impreso, material audiovisual, material informático y telemático, así como la tutoría presencial y telemática.

Otros componentes importantes de la metodología son la estructura y organización 
descritos anteriormente, así como el currículo, la infraestructura y el equipamiento. Consideramos estos puntos como soporte a los principales y no obstante a ello se han considerado de manera exhaustiva en el presente documento.

\section{Evaluación de las licenciatura implanta- da y definición de nuevos programas}

Esta etapa consiste en el diseño de los sistemas de evaluación de los materiales, tutoría, contenidos, docentes y servicios al estudiante. Esta evaluación se realizará a través de cuestionarios y entrevistas con los actores de los procesos, observación directa para los servicios administrativos y tutorías, encuestas a autoridades penitenciarias, empresas y empresarios para medir la aceptación del mercado a los estudiantes. Asimismo coordinar la actualización de currículos y planes de estudios por parte de las respectivas facultades. Esta actividad será desarrollada por la unidad de Investigación y Evaluación.

El siguiente proceso corresponde a la evaluación integral de la metodología propuesta, a los comportamientos del mercado, a los factores coyunturales y los nuevos requerimientos de los alumnos, con el objetivo de elaborar nuevos programas. Esta actividad será desarrollada por la unidad de Marketing mediante estudios de mercado y permitirá corregir y reorientar el rumbo de las actividades desarrolladas a fin de mantener el liderazgo en los servicios de enseñanza y aprendizaje por modalidades a distancia.

\section{DE LOS RECURSOS}

\section{Recursos Humanos}

Asesores y consultores: expertos que evalúan y recomiendan mejoras al programa.

Profesores: encargados del diseño de asignaturas y de la elaboración de los materiales. Asimismo se encargan de la evaluación de los alumnos.

Tutores: encargados de facilitar el aprendizaje de los estudiantes con el diseño y los materiales que elaboraron previamente los profesores.

Personal administrativo: encargados de la gestión operacional del sistema, En el caso del personal directivo se encargan de definición de planes, estrategias y objetivos.

Personal de servicios: encargados de la operatividad y funcionamiento de los locales, infraestructura y recursos educacionales.

Personal de imprenta: encargados de la producción del material impreso.

\section{Recursos de Infraestructura, Materiales y Tecnológicos}

Locales: aulas y oficinas de la sede central y de las unidades descentralizadas.

Bibliotecas: con el números de volúmenes suficiente para atender a los alumnos matriculados. Estas funcionan en las unidades descentralizadas.

Laboratorios de informática: computadoras personales, telecomunicaciones, herramientas de Internet, plataforma educativa de las asignaturas. 
Cafeterías: con la capacidad suficiente para permitir la sociabilización de los alumnos.

Medios de comunicación: para la difusión de las actividades, vinculación con la población, estudiantes, autoridades y empresas.

Materiales de imprenta: materiales de texto para desarrollar.

\section{Administración y financiamiento}

\section{Unidades Descentralizadas}

Las unidades descentralizadas se han seleccionado por las facilidades de infraestructura, capacidad de cobertura e interés por parte de los estudiantes. Estos son: los establecimientos penitenciarios: Reos Primarios "San Jorge" (cercado de Lima) y Mujeres "Santa Mónica" (ubicado en Chorrillos).

\section{CONCLUSIÓN}

La experiencia de instituciones con larga trayectoria en educación superior a distancia nos sugiere considerar la implementación de cursos o licenciaturas específicas de acuerdo con los requerimientos del país, región o institución de cobertura. No es posible copiar o trasladar un modelo de una realidad a otra. Es, en este sentido, necesario impulsar políticas de desarrollo nacional, regional e institucional, implementando programas integrales de formación técnica y profesional.

Es necesario desplegar esfuerzos para la implementación de este programa. Puesto que, de esta manera, los internos podrán lograr obtener un título en una carrera de gran interés para ellos. La Universidad Alas
Peruanas contribuirá a la rehabilitación social de los internos. La posibilidad de comenzar a prepararse, para desempeñar algún oficio posteriormente, actuará como un medio de terapia y le permitirá al interno reincorporarse a la sociedad.

Se requiere la implementación de metodologías de evaluación continua de todos los elementos del programa de educación a distancia en los establecimientos penitenciarios, los alumnos, docentes, recursos, procesos, procedimientos. Esta es la principal vía para asegurar la calidad en el programa a implementarse y en el proceso de enseñanza y aprendizaje del alumno, considerando la situación especial de este último.

Los problemas de gestión deben ser superados en la primera etapa de implementación. Los medios y materiales, así como la información académica y administrativa, deben llegar a los establecimientos penitenciarios en forma oportuna.

Respecto al alumnado, se debe conseguir la identificación plena con la licenciatura a estudiar y con la institución que la ofrece. Cabe resaltar en este punto, que si el alumno obtiene su libertad, podrá continuar con sus estudios en la misma universidad y bajo la misma modalidad.

Resulta necesario e importante desarrollar actividades de integración entre los alumnos y de estos con sus tutores y profesores. Asimismo, ofrecer cursos y actividades de extensión dentro de los establecimientos penitenciarios, motivando la participación de todos los internos. 


\section{BIBLIOGRAFÍA}

GARCÍA ARETIO, Lorenzo. La Educación a Distancia y la UNED. Madrid, Año: 1996 FERNÁNDEZ DE BUJÁN, Federico. La enseñanza universitaria a distancia: una reflexión desde la UNED. Universidad Nacional de Educación a Distancia. Madrid, Año: 2001.

UNIVERSIDAD NACIONAL DE EDUCACIÓN A DISTANCIA. Guía de la Carrera de DERECHO - Curso 2002-2003. Madrid, Año: 2002.

ELBERT, Carlos A. La Universidad en las Cárceles Argentinas. Ediciones Desalma, Buenos Aires, Año: 2000.

FERNÁNDEZ, Adalberto. Modelos de Educación en Centros Penitenciarios. Editorial Humanitas, Barcelona. Año: 1989.

HERRERA BONILLA, Nidia y otros. La Universidad Estatal a Distancia en los Centros Penales de Costa Rica (Ponencia presentada en la Décimo Quinta Conferencia Mundial: "La Educación a Distancia: Desarrollo y Apertura”, Caracas, 1990). Editorial UNED - Costa Rica, Año: 1990.

INSTITUTO UNIVERSITARIO DE EDUCACIÓN A DISTANCIA - IUED. Proyecto Abierto de Investigación: "El alumnado de la UNED en los centros penitenciarios. IUED UNED, Madrid. Año: 2003.

MINISTERIO DE JUSTICIA DEL PERÚ. Constitución Política del Perú (Edición Oficial). Lima, Año: 1993

GARAYCOTT ORELlANA, Norman. Comentarios al Código de Ejecución Penal. Editorial San Marcos, Lima. Año: 2000

OCAMPO RODRÍGUEZ, Esteban. Docencia Universitaria, Teoría y Práctica. Fondo Editorial de la Universidad Alas Peruanas. Lima, Año 2003.

\section{RECURSOS ELECTRÓNICOS:}

www.inpe.gob.pe

Website del Instituto Nacional Penitenciario - INPE 


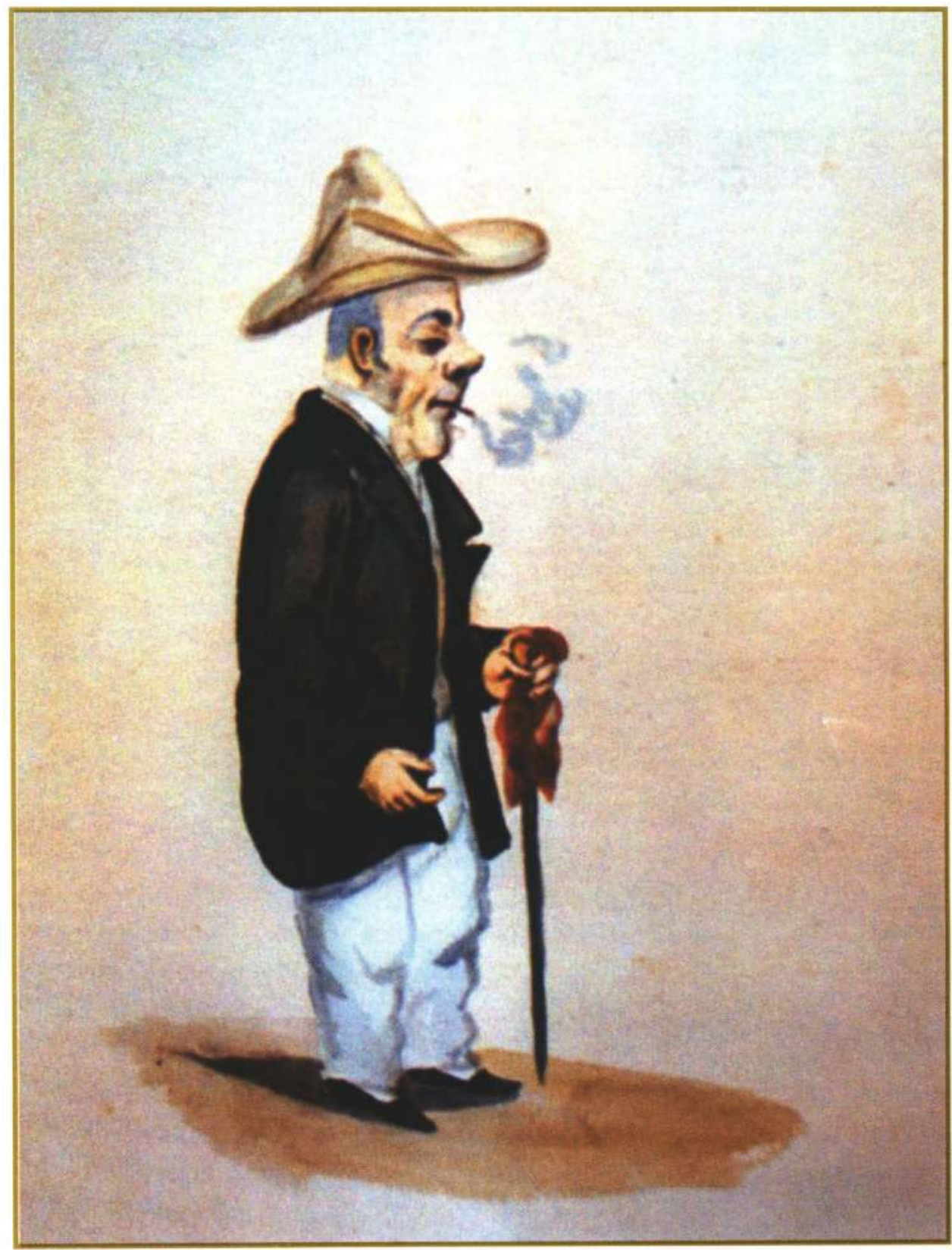

Personaje de la época "Noo Bofetada". 